\title{
Paediatric burn injuries - retrospective evaluation of applied therapeutic management
}

\author{
Jakub Noskiewicz, Małgorzata Rzanny-Owczarzak, Przemysław Mańkowski \\ Department of Paediatric Surgery, Traumatology, and Urology, Poznan University of Medical Sciences, Poznan, Poland
}

\section{ABSTRACT}

Introduction: Each year, nearly $1 \%$ of the world population suffers from burn injuries. However, thermal injuries most often occur in children.

Aim of the study: Burn treatment should consider both local treatment and prevention of existing systemic disturbances. Frequency and importance of the problem requires a revision and optimisation of the existing therapeutic schemes applied for paediatric burns, which constitutes the purpose of this study.

Material and methods: A retrospective analysis was performed on treatment of 310 paediatric patients hospitalised for burns in the years 2010-2017.

Results: In the studied period, 310 patients were hospitalised for burns; the majority of them (66.8\%, 207 patients) were boys. In most cases, hospitalisation was required by children between the first and third year of life (71.9\%, 223 patients). The main cause of burn injuries was contact with hot liquid (98.1\%, 304 patients), primarily in the upper body part. The majority of the patients required only conservative treatment, while split-thickness skin graft (STSG) was performed in $9 \%$ of patients. Early introduction of surgical treatment accelerated wound healing and normalisation of systemic disturbances and correlated with reduced length of stay $(p=0.0019)$. No prevalence of any of the applied professional dressings on the wound healing rate was observed. Substantially, all children required multidrug pain therapy. A low rate of analgesic administration at the pre-hospitalisation stage was recorded.

Conclusions: The vast majority of the patients required only conservative treatment, although the need for introducing surgery should be considered as early as at the first treatment stage. The current condition of burn wound with particular focus on exudation rate and presence of eschar should be the key criterion for the selection of dressing.

KEY WORDS:

burn, child, trauma, wound.

\section{INTRODUCTION}

Each year, nearly $1 \%$ of the world population suffers from burn injuries. However, thermal injuries usually occur in children - in particular up to three years of age. The specifics of burn wounds result from the long period of their morphological formation, in particular of their depth. They form ultimately the third day after thermal injury, i.e. long after the activity of the irritant discontinues. In addition, burn wounds - particularly in children - are frequently concomitant with systemic lesions known as burn disease. The frequency and importance of the problem requires a revision and optimisation of the

\section{ADDRESS FOR CORRESPONDENCE:}

Jakub Noskiewicz, Department of Paediatric Surgery, Traumatology, and Urology, Poznan University of Medical Sciences, 27/33 Szpitalna St., 60-572 Poznan, Poland, e-mail: jnoskiewicz@op.pl 
existing therapeutic schemes applied for burns in patients in developmental age. The purpose of this study is to evaluate the applied schemes and therapeutic management in paediatric burn injuries.

\section{MATERIAL AND METHODS}

A retrospective analysis was performed on the treatment of 310 paediatric patients hospitalised for isolated thermal injury in the Department of Paediatric Surgery in the years 2010-2017. The performed analysis used, among others, treatment records of patients with burns drawn up for the purposes of the study. The records contained information on the circumstances of injury, procedures implemented on-site, and the course of specialisation. The course of therapeutic management with particular focus on local treatment of burn wounds and introduced analgesic therapy was evaluated. The acquired data were analysed using descriptive statistical methods and tools, adopting a significance level of $p<0.05$. Consent for performing the studies was issued by the Bioethical Committee (no. 175/17).

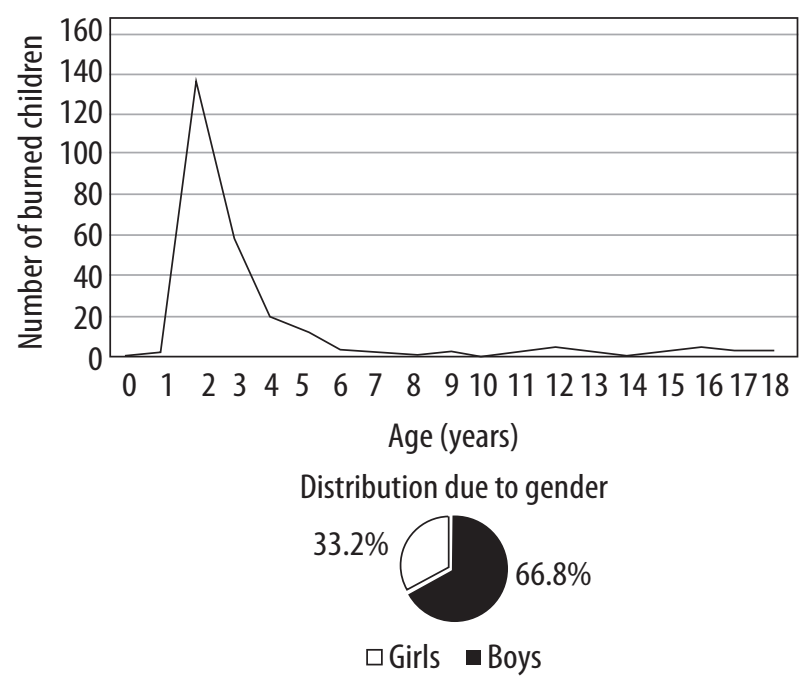

FIGURE 1. Distribution of burns in children due to age and gender

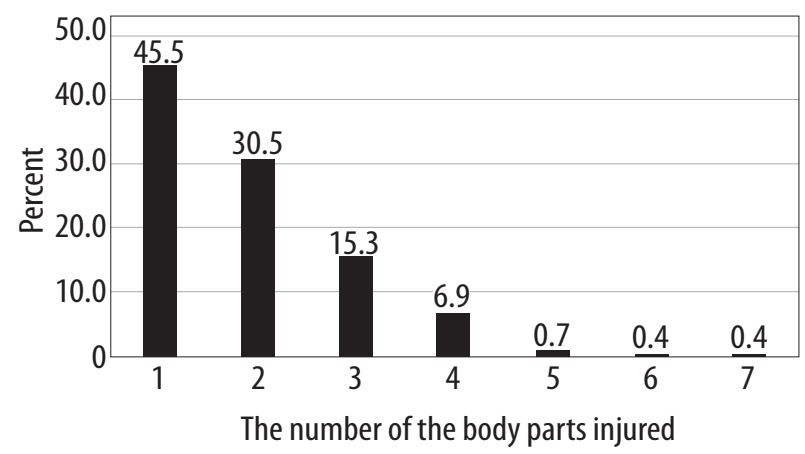

FIGURE 2. Distribution of burns in children due to the number of injuries of body regions

\section{RESULTS}

In the years 2010-2017, 310 paediatric patients were hospitalised for thermal injury, comprising $66.8 \%$ boys (207 patients) and 33.2\% girls (103 patients). The median age of patients was one year (age range from 4 months to 18 years). In the vast majority hospitalisation was required in children between the first and third year of age. This age group accounted for $71.9 \%$ of all hospitalisations (223 patients) (Fig. 1). The main cause of burns was thermal injury resulting from contact with hot liquid or an object (98.1\%, 304), occurring in most cases at home. The following burn injuries were also recorded: electrical (1.3\%, $4)$, chemical $(0.3 \%, 1)$, and sunburn $(0.3 \%, 1)$. On average, approximately $10 \%$ of body surface was injured. The most common body parts injured were: upper limbs $(63.2 \%$, $196)$, the chest $(44.8 \%, 139)$, and the head and neck $(30.6 \%$, $95)$ with dominating second-degree burns. More than half $(54.5 \%, 169)$ of the children had at least two body parts injured (Fig. 2). Thermal injuries, in particular the extensive ones, occurred primarily in the afternoon hours.

More than half $(57.1 \%)$ of burn wounds were cooled with water by the parents or carers directly after thermal injury. Average cooling time was 10 minutes. Immediate cooling of thermally damaged tissues significantly reduced length of stay ( $p=0.0314)$ from eight to five days on average. In the case of $34.3 \%$ of patients, first aid after injury was provided by the Emergency Response Team (ERT). On one hand, its activities consist of wound treatment, primarily with the use of hydrogel dressings. On the other hand, the ERT immediately secures the vital functions, applies initial fluid therapy and analgesia and transport to a paediatric burn treatment centre, whilst the analgesics were administered only at every fourth medical intervention and were primarily the opioids - in most cases morphine (66.7\%). Parents or carers administered analgesics, usually ibuprofen, to children with burn injuries on a sporadic basis.

On admission to hospital, the general condition and burn injury of each child was assessed. The wounds were secured upon cleaning, in most cases with hydrocolloid dressings $(96.1 \%, 298)$. At the same time, systemic treatment was performed with particular focus on: fluid therapy, analgesia, and nutritional treatment. Emergency surgery (escharotomy, excision of eschar) within 48 hours of thermal injury was required in two patients $(0.6 \%)$. For $3.2 \%$ (10) of the patients it was necessary to perform central venous access at the first stage of burn disease management. Final evaluation of depth and surface of burn injury was performed within 72 hours of the beginning of hospitalisation. After removing the dressings and excision of eschar, the patients were qualified for conservative or surgical treatment. Implementation of non-surgical management required application of hydrocolloid, foam, or hydrofiber dressings - containing, among others, silver. Selection of 
the type of dressing depended on the volume of exudation within the burn wound and of the excised eschar. No impact of the individual types of dressings on length of stay and wound healing rate was observed (Fig. 3). Burn injuries in the head or groin area were treated without dressings. 1.9\% (6) of children required enzyme supplementation during eschar excision by means of locally supplied clostridiopeptidase A. On average, dressings were changed twice per hospitalisation. Conservative treatment alone was introduced in $91.0 \%$ (282) of patients. Average length of hospital stay in this group was six days.

Nine per cent (28) of children with burns required surgery. In most cases, the procedure consisted of excision of eschar from wounds and autologous free-tissue transfer of split-thickness skin grafted primarily from thigh areas. In this group, about a half of the cases $(53.6 \%$, 16) were qualified to the surgical procedure in a deferred mode - after several days of conservative treatment attempts that failed to bring satisfactory effects. Early introduction of surgery accelerated wound healing and recovery from systemic disturbances and therefore significantly correlated with reduced length of stay $(p=0.0019)$. The time period between surgery and discharge from hospital was no different for both groups of patients - with early and deferred surgery $(p=0.1328)$. Also, no significant differences in the graft healing depending on the applied dressing or its fixation (no edges fixed, absorbable sutures, fibrin glue) were observed. On the other hand, better graft healing and therefore local treatment acceleration was recorded after applying the vacuum dressing within the burn wound covered with an intermediate-thickness graft (suction pressure up to $60 \mathrm{mmHg}$ ). The use of negative pressure wound therapy reduced the average time of hospitalisation by more than one-third (33.8\%). In the group of patients with surgery, the average length of stay was 16 days, whereas the period between the procedure and discharge from hospital was 8 days (Fig. 4).

Intravenous paracetamol was the foundation of pain management in paediatric patients with burn injuries. The frequency of its use increased in subsequent years ( $77.0 \%$ in $2010,100.0 \%$ in 2017). On average, half of the patients required strong opioids, which were also effectively used to ensure adequate analgesia during dressing changes. The least commonly administered drugs included weak opioids (11.0\%) and ketoprofen (3.9\%) (Fig. 5). More than half of the children with burns $(68.1 \%, 211)$ required at least two analgesics during hospitalisation to achieve an adequate analgesic effect. In $7.1 \%$ (22) of patients it was necessary to administer at least four different analgesics. This polyanalgesia enabled successful elimination of pain. Median pain level during hospitalisation, excluding dressing change, was 0 on a numeric pain rating scale (maximal pain - 7). With regard to shorter length of stay and introduction of the advanced wound dressing methods, total doses of analgesics included during hospitalisation were significantly reduced. No significant

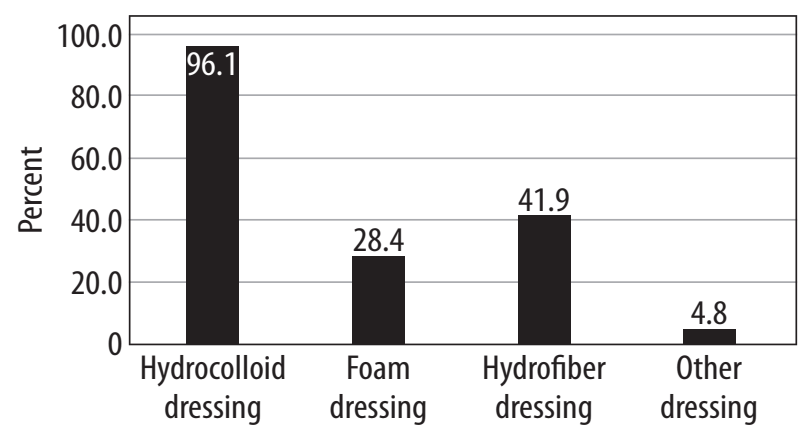

FIGURE 3. Frequency of application of different types of dressings in the treatment of burn wounds in children

or permanent adverse effects of this type of pain therapy were recorded.

\section{DISCUSSION}

Burn injuries are suffered primarily by younger children up to preschool age. This is correlated with significant physical activity resulting from intensive psychomotor development in this period of life and simultaneous lack of criticism of actions taken and an inability to prefigure potential threats. Injuries occur mostly at home by pulling down dishes containing hot liquids. This group is slightly dominated by boys, although, according to Moehrlen et al., their domination increases with age. On the other hand, Alnababtah and Khan believe that people living in social housing are at greater risk of burns. According to Verey et al., the vast majority of injuries, in particular the extensive ones, occur mainly in the afternoon hours. A similar regularity was observed by the authors of this study. Kemp et al. emphasise additionally that up to $25.0 \%$ of burns can be correlated with abuse. The probability of intentional injury can be estimated with the use of a test proposed by these researchers. Detailed knowledge on the structure of populations with burn injuries and the most frequent injury circumstances or schemes is crucial and helpful in planning and carrying out prophylactic activities reducing the risk of paediatric burns. Due to the frequent occurrence of burns in children, it seems important to organise prophylactic actions against thermal injuries. Such actions should pay attention to the necessity of eliminating factors or behaviours that increase the risk of child burns - especially in the home, where children are most often burned. At the same time, during such actions, the elements of first aid for the burned child should be presented. Unfortunately, the problem of burns is not often discussed in the media [1-5].

Implementation of adequate procedure directly after injury is one of the key elements of burn management. Immediate cooling of wound, in most cases by the parents, that can significantly decrease the depth of injured tissues and therefore reduce treatment length, plays an important role here. According to numerous sources, cooling is performed in up to $90.0 \%$ of children with 

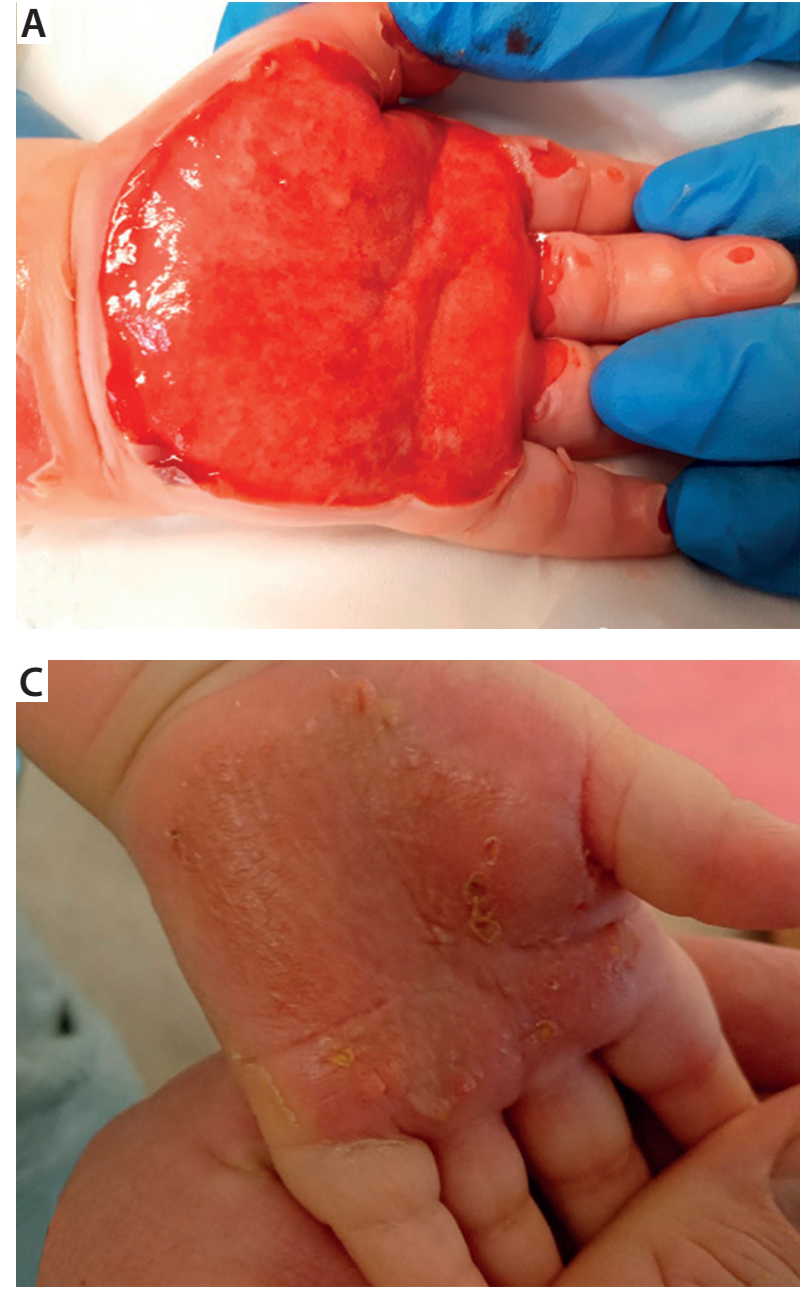

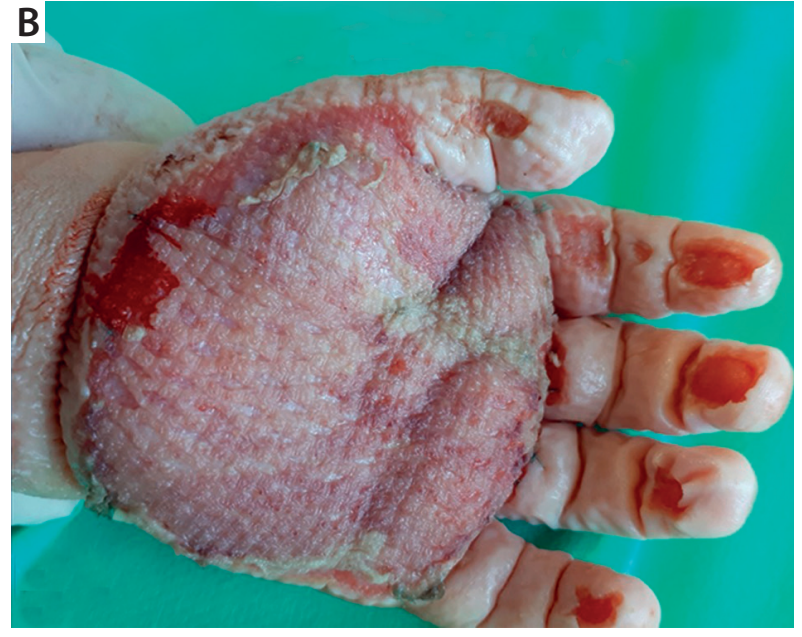

FIGURE 4. Burn in an infant: A) partially cleaned of necrosis burn wound before skin grafting, B) wound two days after split-thickness skin transplantation, $\mathrm{C}$ ) healed wound two weeks after skin transplantation

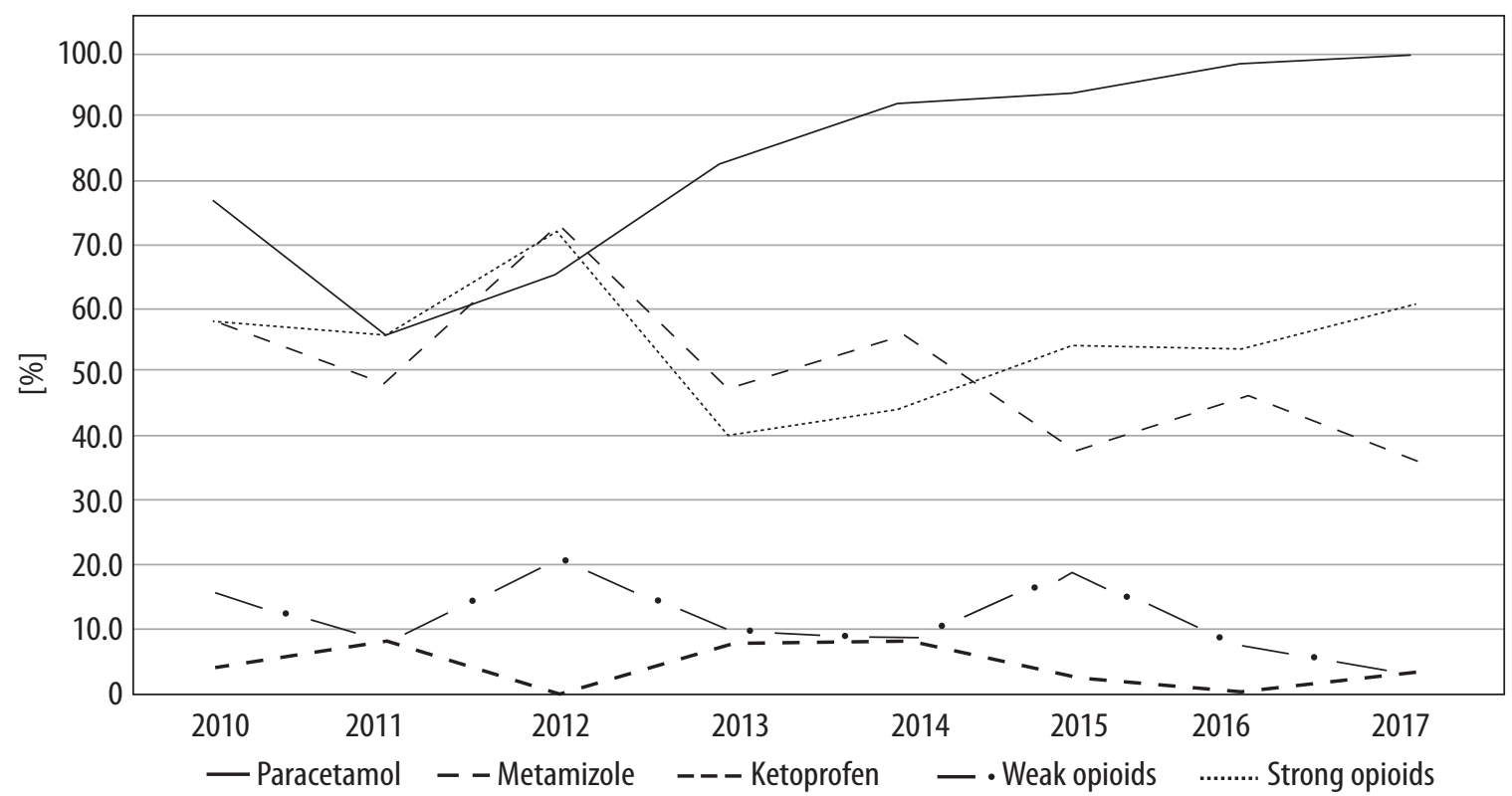

FIGURE 5. The use of analgesics in the treatment of burns in children in 2010-2017

burns, i.e. much more frequently than in the studied population. According to the authors of this publication, this results from the lack of sufficient knowledge and skills to provide first aid to burned children in Polish society. It should also be emphasised that cooling the wound has a significant analgesic effect, especially when there is no possibility to administer analgesics $[6,7]$.

Pain suffered from burnt tissues is one of the strongest negative sensations for humans. Therefore, implementation of an adequate analgesic procedure is a key element 
of treating children after thermal injury. A relatively low percentage of the patients supplied with analgesics directly after the injury, in particular by the Emergency Response Teams, is noticeable. The issue of inadequate pain assessment and rare administration of analgesics - specifically opioids - at the pre-hospitalisation stage is also brought up by Browne et al. The lack of proper analgesia and poorly conducted fluid therapy are the main weak points in the treatment of children with thermal injuries at the pre-hospitalisation stage. Therefore, it would be necessary to create guidelines for prehospital treatment of burned children, with particular attention to analgesic treatment. The scheme of further analgesic management during hospitalisation depends mostly on the internal guidelines developed in the burn treatment centres. At present, there are no general and commonly accepted pain management standards in children after thermal injury, although key roles are played by: paracetamol, metamizole, and opioids (primarily morphine). There are also no harmonised recommendations for sedatives and analgesics administered during dressing changes [7-9].

The development of advanced dressings was a milestone in burn wound treatment. Such dressings create conditions within the wound that foster the healing process. Silver dressings are particularly useful. Their application reduces the risk of wound infection, fosters the epithelialisation process, shortens the length of treatment and hospitalisation, and decreases scarring, thus enabling a better final aesthetic effect. The above is confirmed by the studies of Schiestl et al. and Lau et al. On the other hand, there is no prevalence of any type of dressing observed. The exudation rate within the burn wound and volume of eschar seem to be the main selection criteria [10-12].

The therapeutic standard for deep burn wounds is autologous free tissue transfer of split-thickness graft onto the previously cleaned burn wound. The other available options for skin loss (skin equivalents, keratinocyte cultures, xenografts) are less common. Nowadays, the focus is on advantages brought by immediate surgical interventions in full-thickness burns. On one hand, this procedure accelerates wound healing, whereas on the other hand it inhibits progression of burn disease and therefore shortens the length of treatment and hospitalisation. The graft healing length can be reduced by using vacuum dressings, provided that the authors of the publication - in contrast to Koehler et al. - believe that post-grafting placement of a single post-grafting vacuum dressing and keeping it for at least 48 hours is sufficient [13].

An important element in the treatment of burns is also post-operative procedure. Proper management of the burned child after discharge from the hospital has a significant impact on the final effect of the wound healing process. It allows the appropriate aesthetic effect to be obtained and reduces the risk of functional disorders (contractures caused by scars). This procedure consists mainly of compression therapy (also using specially tailored compressed clothes) and the use of collagen or silicone agents to limit scarring. Reducing contact with ultraviolet radiation is also important.

\section{CONCLUSIONS}

Paediatric burn management is a complex process. It requires simultaneous local treatment and introduction of an adequate systemic procedure. The majority of the patients require only conservative treatment, although the need to introduce surgery should be considered as early as at the first treatment stage.

The current condition of the burn wound with particular focus on the exudation rate and presence of eschar should be the key criteria for the selection of dressing.

Adequate pain therapy, which should be commenced at the pre-hospitalisation stage, constitutes an important component of paediatric burn injury management.

\section{DISCLOSURE}

The authors declare no conflict of interest.

\section{REFERENCES}

1. Moehrlen T, Szucs T, Landolt MA, et al. Trauma mechanisms and injury patterns in pediatric burn patients. Burns 2018; 44: 326-334.

2. Alnababtah K, Khan S. Socio-demographic factors which significantly relate to the prediction of burns severity in children. Int J Burns Trauma 2017; 7: 56-63.

3. Verey F, Lyttle MD, Lawson Z, et al. When do children get burnt? Burns 2014; 40: 1322-1328.

4. Kemp AM, Hollén L, Emond AM, et al. Raising suspicion of maltreatment from burns: Derivation and validation of the BuRN-Tool. Burns 2018; 44: 335-343.

5. Wang S, Li D, Shen C, et al. Epidemiology of burns in pediatric patients of Beijing City. BMC Pediatr 2016; 16: 166.

6. Baartmans MG, de Jong AE, van Baar ME, et al. Early management in children with burns: Cooling, wound care and pain management. Burns 2016; 42: 777-782.

7. Haines E, Fairbrother H. Optimizing emergency management to reduce morbidity and mortality in pediatric burn patients. Pediatr Emerg Med Pract 2015; 12: 21-23.

8. Browne LR, Studnek JR, Shah MI, et al. Prehospital Opioid Administration in the Emergency Care of Injured Children. Prehosp Emerg Care 2016; 20: 59-65.

9. Singleton A, Preston RJ, Cochran A. Sedation and analgesia for critically ill pediatric burn patients: the current state of practice. J Burn Care Res 2015; 36: 440-445.

10. Schiestl C, Meuli M, Trop M, Neuhaus K. Management of burn wounds. Eur J Pediatr Surg 2013; 23: 341-348.

11. Lau CT, Wong KK, Tam P. Silver containing hydrofiber dressing promotes wound healing in paediatric patients with partial thickness burns. Pediatr Surg Int 2016; 32: 577-581.

12. Józsa G1, Tóth E1, Juhász Z. New dressing combination for the treatment of partial thickness burn injuries in children. Ann Burns Fire Disasters 2017; 30: 43-46.

13. Koehler S, Jinbo A, Johnson S, et al. Negative pressure dressing assisted healing in pediatric burn patients. J Pediatr Surg 2014; 49: 1142-1145. 